\title{
DETERMINATION OF BIOACTIVE COMPONENTS OF BARLERIA COURTALLICA NEES (ACANTHACEAE) BY GAS CHROMATOGRAPHY-MASS SPECTROMETRY ANALYSIS
}

\author{
PONMATHI SUJATHA A ${ }^{1}$, MICHAEL EVANJALINE $\mathrm{R}^{1}$, MUTHUKUMARASAMY $\mathrm{S}^{2}$, MOHAN VR ${ }^{1 *}$ \\ ${ }^{1}$ Research Department of Botany, Ethnopharmacology Unit, V.0. Chidambaram College, Tuticorin - 628 008, Tamil Nadu, India. \\ ${ }^{2}$ Department of Botany, Sri K.G.S. Arts College, Srivaikuntam - 628 619, Tamil Nadu, India. Email: vrmohanvoc@gmail.com
}

Received: 20 February 2016, Revised and Accepted: 24 March 2017

\section{ABSTRACT}

Objective: The present investigation was carried out to determine the possible bioactive components of stem, root, and leaf of Barleria courtallica Nees using a gas chromatography-mass spectrometry (GC-MS).

Methods: The phytocomponents of the ethanol extracts of stem, root, and leaf of B. courtallica were investigated using PerkinElmer GC-MS, while the mass spectra of the compounds found in the extracts were matched with the National Institute of Standards and Technology version II library.

Results: 25, 23, and 28 compounds were identified in the ethanol extracts of stem, root, and leaf of $B$. courtallica, respectively. The prevailing compounds of stem were $\beta$-sitosterol (20.27\%), stigmasterol (17.07\%), eicosane, 7-hexyl- (6.64\%), 3,7,11,15-tetramethyl-2-hexadecan-1-ol (5.97\%), and tetracosane, 11-decyl- (5.91\%). The major constituents recorded from root extract of $B$. courtallica were $\beta$-sitosterol (22.94\%), stigmasterol (20.17\%), urs-12-en-28-oic acid, 3-hydroxy-, methyl ester, (3ß)- (18.42\%), and eiosane, 7-hexyl- (7.06\%). The prevailing compounds of leaf were 3,7,11,15- tetramethyl-2-hexadecan-1-ol (34.42\%), phytol (14.18\%), $\beta$-sitosterol (12.71\%), squalene (11.25\%), stigmasterol (8.15\%), phytol acetate (6.53\%).

Conclusions: From the results, it is evident that B. courtallica contains various bioactive compounds and is recommended as a plant of phytopharmaceutical importance.

Keywords: Barleria courtallica, Bioactive components, Phytol, Acetate, Squalene.

(C) 2017 The Authors. Published by Innovare Academic Sciences Pvt Ltd. This is an open access article under the CC BY license (http://creativecommons. org/licenses/by/4. 0/) DOI: http://dx.doi.org/10.22159/ajpcr.2017.v10i6.18035

\section{INTRODUCTION}

Medicinal plants besides therapeutic agents are also a big source of information for a wide variety of chemical constituents which could be developed as drugs with precise selectively. These are the reservoirs of potentially useful chemical compounds which could serve as newer leads and clues for modern drug design [1]. The most important of these bioactive constituents of plants are alkaloids, tannins, flavonoids, glycosides, saponins, and phenolic compounds [2]. Correlation between the phytoconstituents and the bioactivity of the plant is desirable to know for the synthesis of compounds with specific activities to treat various health ailments and chronic diseases as well [3].

A knowledge of the chemical constituents of plants is desirable not only for the discovery of therapeutic agents but also because such information may be of great value in disclosing new sources of economic phytocompounds for the synthesis of complex chemical substances and for discovering the act was significance of folkloric remedies [4]. Hence, a thorough validation of the herbal drugs has emerged as a new branch of science emphasizing and prioritizing the standardization of the natural drugs and products because several of the phytochemicals have complementary and overlapping mechanism of action. Mass spectrometry (MS), coupled with chromatographic separations such as gas chromatography (GC/MS), is normally used for direct analysis of components existing in traditional medicines and medicinal plants. In recent years, GC-MS studies have been increasingly applied for the analysis of medicinal plants as this technique has proved to be a valuable method for the analysis of non-polar components and volatile essential oil, fatty acids, lipids, and alkaloids [5-7].

Genus Barleria belongs to the family Acanthaceae. Whole-plant extract Barleria contains a number of active compounds such as alkaloids, terpenes, flavonoids, glycosides, lignins, and phenolics, which have shown potent therapeutic activities against several diseases [8-11]. Barleria also shows various pharmacological effects such as antimicrobial, anthelmintic, antifertility, antioxidant, antidiabetic, antiarthritic, hepatoprotective, diuretic, cytoprotective, antidiarrheal, analgesic, antileukemic, anti-inflammatory, and hypoglycemic properties without any toxic effects $[12,13]$. Taking into consideration of the medicinal importance of the plant, the ethanol extract of Barleria courtallica was analyzed for GC-MS. Till now, the investigation of phytocomponents by GC-MS has not been done on B. courtallica Nees. The present study was carried out to identify some of the phytocomponents present in the ethanol extracts of $B$. courtallica (stem, root, and leaf) by GC-MS technique, to ascertain the medicinal properties of the plant.

\section{METHODS}

\section{Collection of plant sample}

The whole plant of B. courtallica Nees was collected from Agasthyamala Biosphere Reserve, Western Ghats, Tamil Nadu. The plant was identified with the help of local flora and authenticated in Botanical Survey of India, Southern Circle, Coimbatore, Tamil Nadu. The voucher specimens (VOCB3336) were preserved in the Ethnopharmacology Unit, Research Department of Botany, V.O. Chidambaram College, Tuticorin, Tamil Nadu.

\section{Preparation of plant extract}

Stem, root, and leaf of B. courtallica were cleaned, shade-dried, and pulverized to powder in a mechanical grinder. Required quantity of each powder samples (stem, root, and leaf) was weighed and transferred to stoppered flask separately and treated with ethanol until the powder is fully immersed. The flask was shaken every hour for the first $6 \mathrm{hrs,}$ 
and then it was kept aside and again shaken after $24 \mathrm{hrs}$. This process was repeated for 3 days, and then the extracts were filtered. The extracts were collected and evaporated to dryness using vacuum distillation unit. The final residue thus obtained was then subjected to GC-MS analysis.

\section{GC-MS analysis}

GC-MS analysis of ethanol extract was performed with GC Clarus 500 PerkinElmer system and GC interfaced to an MS equipped with Elite-1 fused silica capillary column $(30 \times 0.25 \mathrm{~mm} 1 \mathrm{D} \times 1 \mathrm{um} \mathrm{df}$, composed of $100 \%$ dimethyl polysiloxane). For GC-MS detection, electron ionization system with ionizing energy of $70 \mathrm{eV}$ was used. Helium gas (99.999\%) was used as the carrier gas at constant flow rate $1 \mathrm{ml} /$ minute and an injection volume of $2 \mu \mathrm{l}$ was employed (split ratio of 10:1); injector temperature $250^{\circ} \mathrm{C}$; ion source temperature $280^{\circ} \mathrm{C}$. The oven temperature was programmed from $110^{\circ} \mathrm{C}$ (isothermal for 2 minutes) with an increase of $10^{\circ} \mathrm{C} /$ minutes, to $200^{\circ} \mathrm{C}$, then $5^{\circ} \mathrm{C} /$ minutes to $280^{\circ} \mathrm{C}$, ending with 9 minutes isothermal at $2800 \mathrm{C}$. Mass spectra were taken at $70 \mathrm{eV}$, a scan interval of 0.5 seconds and fragments from 45 to $450 \mathrm{Da}$. Total GC running time was 36 minutes. The relative \% amount of each component was calculated by comparing its average peak area to the total areas; software adopted to handle mass spectra and chromatograms was a turbomass. Interpretation on mass spectrum of GC-MS was done using the database of National Institute of Standard and Technology (NIST) having more than 62,000 patterns. The mass spectrum of the unknown component was compared with the spectrum of the known components stored in the NIST library. The name, molecular weight, and structure of the components of the test materials were ascertained.

\section{RESULTS}

The compounds present in the ethanol extract of stem, root, and leaf of B. courtallica were identified by GC-MS analysis (Figs. 1-3).
The active principles with their retention time, molecular formula, molecular weight, peak area \%, and molecular structures are presented in Tables 1-3.

25,23 , and 28 compounds were identified in the ethanol extracts of stem, root, and leaf of $B$. courtallica, respectively. The prevailing compounds of stem were $\beta$-sitosterol (20.27\%), stigmasterol (17.07\%), eicosane, 7-hexyl- (6.64\%), 3,7,11,15-tetramethyl-2-hexadecan-1-ol (5.97\%), tetracosane, 11-decyl- (5.91\%), dodecyl cis-9,10-epoxyoctadecanoate (5.84\%), 9-octadecenamide, (Z)- (5.47\%), dibutyl phthalate (5.08\%), vitamin E (4.67\%), squalene (4.32\%), 2-methylhexacosane $(4.20 \%)$, phytol (3.54\%), and Z,Z-3,15-octadecadien-1-ol acetate $(3.26 \%)$. The major constituents recorded from root extract of $B$. courtallica were $\beta$-sitosterol (22.94\%), stigmasterol (20.17\%), urs-12-en-28-oic acid, 3-hydroxy-, methyl ester, (3ß)-(18.42\%), eiosane, 7-hexyl- (7.06\%), 9-octadecenamide, (Z)- (4.82\%), 2-methyl hexacosane (3.54\%), dodecyl cis-9,10-epoxyoctadecanoate (3.15\%), androstane-11, 17-dione, 3-[(trimethylsilyl)oxy]-,17-[0-(phenylmethyl)oxime], (3 $\alpha$, $5 \alpha)-(2.89 \%)$, dibutyl phthalate $(2.83 \%)$, and heptacosane $(2.81 \%)$. The prevailing compounds of leaf were 3,7,11,15-tetramethyl-2hexadecan-1-ol (34.42\%), phytol (14.18\%), $\beta$-sitosterol $(12.71 \%)$, squalene $(11.25 \%)$, stigmasterol $(8.15 \%)$, phytol acetate $(6.53 \%)$, vitamin E (3.13\%), tetracosane, 11-decyl-(1.69\%), and lupeol $(1.60 \%)$. Table 4 lists the various phytoconstituents which contribute to the biological activity of ethanol extracts of stem, root, and leaf of B. courtallica.

\section{DISCUSSION}

The results pertaining to GC-MS analysis led to the identification of number of compounds from the GC fraction of the ethanol extracts of B. courtallica stem, root, and leaf. These compounds were identified

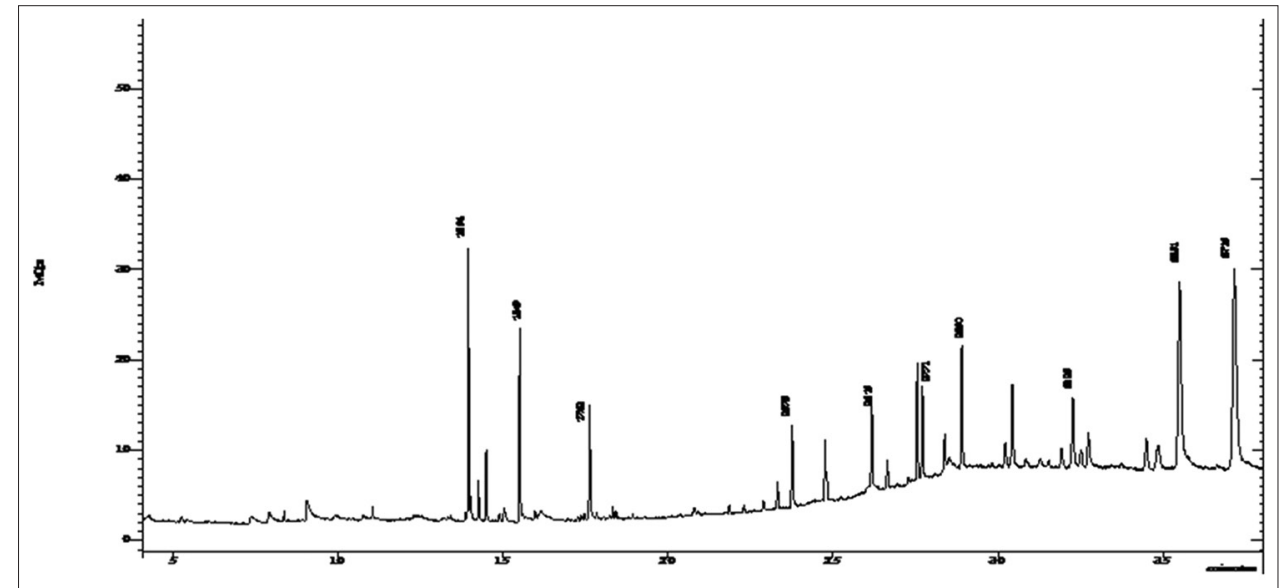

Fig. 1: Gas chromatography-mass spectrometry chromatogram of the ethanol extract of stem of Barleria courtallica



Fig. 2: Gas chromatography-mass spectrometry chromatogram of the ethanol extract of root of Barleria courtallica 


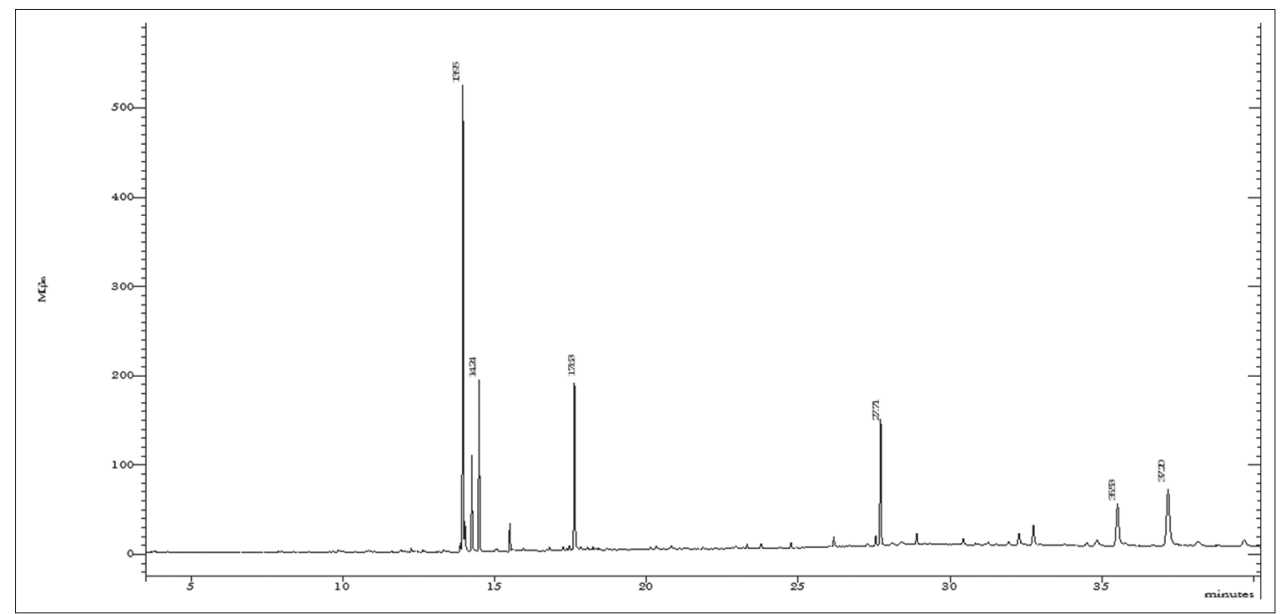

Fig. 3: Gas chromatography-mass spectrometry chromatogram of the ethanol extract of leaf of Barleria courtallica

Table 1: Phytocomponents detected in stem of $B$. courtallica

\begin{tabular}{|c|c|c|c|c|c|}
\hline RT & Name of the compound & $\begin{array}{l}\text { Molecular } \\
\text { formula }\end{array}$ & $\begin{array}{l}\text { Molecular } \\
\text { weight }\end{array}$ & $\begin{array}{l}\text { Peak area } \\
\%\end{array}$ & Molecular structure \\
\hline 7.38 & p-Cymen-7-ol & $\mathrm{C}_{10} \mathrm{H}_{14} \mathrm{O}$ & 150 & 0.12 & \\
\hline 7.92 & $\begin{array}{l}\text { 1,4-methanoazulene-9-methanol, } \\
\left.\text { decahydro-4,8,8-trimethyl-, [1S- }\left(1 \alpha, 3 \mathrm{a} \beta, 4 \alpha, 8 \mathrm{a} \beta, 9 \mathrm{R}^{*}\right)\right] \text { - }\end{array}$ & $\mathrm{C}_{15} \mathrm{H}_{26} \mathrm{O}$ & 222 & 0.13 & \\
\hline 8.39 & 4,7,7-Trimethylbicyclo[2.2.1]heptan-2-one 0-allyloxime & $\mathrm{C}_{13} \mathrm{H}_{21} \mathrm{NO}$ & 207 & 0.20 & \\
\hline 9.04 & $\alpha$-D-Glucopyranose, 4-O- $\beta$-D-galactopyranosyl- & $\mathrm{C}_{12} \mathrm{H}_{22} \mathrm{O}_{11}$ & 342 & 0.41 & \\
\hline 11.03 & Diethyl phthalate & $\mathrm{C}_{12} \mathrm{H}_{14} \mathrm{O}_{4}$ & 222 & 0.30 & \\
\hline 13.94 & 3,7,11,15-Tetramethyl-2-hexadecen-1-ol & $\mathrm{C}_{20} \mathrm{H}_{40} \mathrm{O}$ & 296 & 5.97 & \\
\hline 14.24 & 5-Nonadecen-1-ol & $\mathrm{C}_{19} \mathrm{H}_{38} \mathrm{O}$ & 282 & 0.96 & \\
\hline 14.48 & Phytol acetate & $\mathrm{C}_{22} \mathrm{H}_{42} \mathrm{O}_{2}$ & 338 & 1.62 & \\
\hline 15.49 & Dibutyl phthalate & $\mathrm{C}_{16} \mathrm{H}_{22} \mathrm{O}_{4}$ & 278 & 5.08 & \\
\hline 17.62 & Phytol & $\mathrm{C}_{20} \mathrm{H}_{40} \mathrm{O}$ & 296 & 3.54 & \\
\hline 18.31 & 9,12-Octadecadienoic acid (Z, Z)-, methyl ester & $\mathrm{C}_{19} \mathrm{H}_{34} \mathrm{O}_{2}$ & 294 & 0.28 & \\
\hline
\end{tabular}


Table 1: (Continued...)

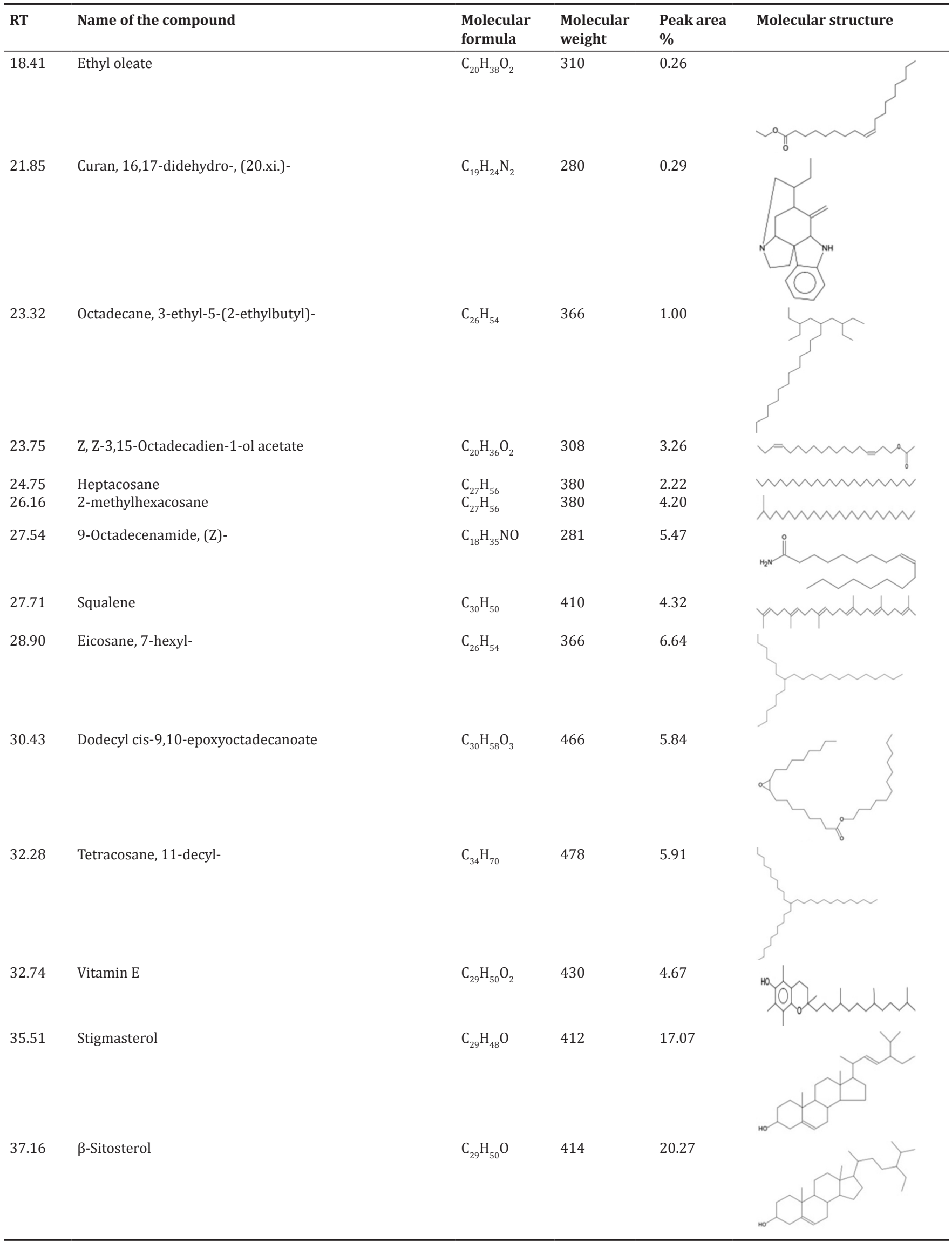

B. courtallica: Barleria courtallica 
Table 2: Phytocomponents detected in root of $B$. courtallica

\begin{tabular}{|c|c|c|c|c|c|}
\hline RT & Name of the compound & $\begin{array}{l}\text { Molecular } \\
\text { formula }\end{array}$ & $\begin{array}{l}\text { Molecular } \\
\text { weight }\end{array}$ & $\begin{array}{l}\text { Peak area } \\
\%\end{array}$ & Molecular structure \\
\hline 7.38 & p-Cymen-7-ol & $\mathrm{C}_{10} \mathrm{H}_{14} \mathrm{O}$ & 150 & 0.14 & \\
\hline 7.87 & $\begin{array}{l}\text { Cyclohexene, 4-ethenyl-4-methyl-3-(1-methylethenyl)- } \\
\text { 1-(1-methylethyl)-, (3R-trans)- }\end{array}$ & $\mathrm{C}_{15} \mathrm{H}_{24}$ & 204 & 0.91 & \\
\hline 8.36 & 4,7,7-trimethylbicyclo[2.2.1]heptan-2-one 0-allyloxime & $\mathrm{C}_{13} \mathrm{H}_{21} \mathrm{NO}$ & 207 & 0.27 & \\
\hline 9.04 & $\alpha$-D-glucopyranose, 4-O- $\beta$-D-galactopyranosyl- & $\mathrm{C}_{12} \mathrm{H}_{22} \mathrm{O}_{11}$ & 342 & 0.54 & \\
\hline 11.03 & Diethyl phthalate & $\mathrm{C}_{12} \mathrm{H}_{14} \mathrm{O}_{4}$ & 222 & 0.52 & \\
\hline 13.94 & 3,7,11,15-tetramethyl-2-hexadecen-1-ol & $\mathrm{C}_{20} \mathrm{H}_{40} \mathrm{O}$ & 296 & 0.41 & \\
\hline 14.48 & Phytol, acetate & $\mathrm{C}_{22} \mathrm{H}_{42} \mathrm{O}_{2}$ & 338 & 0.18 & \\
\hline 15.49 & Dibutyl phthalate & $\mathrm{C}_{16} \mathrm{H}_{22} \mathrm{O}_{4}$ & 278 & 2.83 & \\
\hline 18.42 & Ethyl oleate & $\mathrm{C}_{20} \mathrm{H}_{38} \mathrm{O}_{2}$ & 310 & 0.37 & \\
\hline 21.85 & Curan, 16,17-didehydro-, (20.xi.)- & $\mathrm{C}_{19} \mathrm{H}_{24} \mathrm{~N}_{2}$ & 280 & 0.20 & \\
\hline 23.32 & Octadecane, 3-ethyl-5-(2-ethylbutyl)- & $\mathrm{C}_{26} \mathrm{H}_{54}$ & 366 & 1.00 & \\
\hline 23.76 & Z, Z-3,15-octadecadien-1-ol acetate & $\mathrm{C}_{20} \mathrm{H}_{36} \mathrm{O}_{2}$ & 308 & 1.69 & \\
\hline
\end{tabular}


Table 2: (Continued...)

\begin{tabular}{|c|c|c|c|c|c|}
\hline RT & Name of the compound & $\begin{array}{l}\text { Molecular } \\
\text { formula }\end{array}$ & $\begin{array}{l}\text { Molecular } \\
\text { weight }\end{array}$ & $\begin{array}{l}\text { Peak area } \\
\%\end{array}$ & Molecular structure \\
\hline 24.75 & Heptacosane & $\mathrm{C}_{27} \mathrm{H}_{56}$ & 380 & 2.81 & mmmmm \\
\hline 26.16 & 2-methylhexacosane & $\mathrm{C}_{27} \mathrm{H}_{56}$ & 380 & 3.54 & \\
\hline 27.54 & 9-octadecenamide, (Z)- & $\mathrm{C}_{18} \mathrm{H}_{35} \mathrm{NO}$ & 281 & 4.82 & \\
\hline 27.71 & Squalene & $\mathrm{C}_{30} \mathrm{H}_{50}$ & 410 & 2.82 & \\
\hline 28.52 & Urs-12-en-28-oic acid, 3-hydroxy-, methyl ester, (3ß)- & $\mathrm{C}_{31} \mathrm{H}_{50} \mathrm{O}_{3}$ & 470 & 18.42 & \\
\hline 28.90 & Eicosane, 7-hexyl- & $\mathrm{C}_{26} \mathrm{H}_{54}$ & 366 & 7.06 & \\
\hline 30.43 & Dodecyl cis-9,10-epoxyoctadecanoate & $\mathrm{C}_{30} \mathrm{H}_{58} \mathrm{O}_{3}$ & 466 & 3.15 & \\
\hline 32.25 & Tetracosane, 11-decyl- & $\mathrm{C}_{34} \mathrm{H}_{70}$ & 478 & 2.32 & \\
\hline 34.85 & $\begin{array}{l}\text { Androstane-11,17-dione, 3-[(trimethylsilyl)oxy]-, } \\
\text { 17-[0-(phenylmethyl)oxime], }(3 \alpha, 5 \alpha) \text { - }\end{array}$ & $\mathrm{C}_{29} \mathrm{H}_{43} \mathrm{NO}_{3} \mathrm{Si}$ & 481 & 2.89 & \\
\hline 35.51 & Stigmasterol & $\mathrm{C}_{29} \mathrm{H}_{48} \mathrm{O}$ & 412 & 20.17 & \\
\hline 37.17 & $\beta$-Sitosterol & $\mathrm{C}_{29} \mathrm{H}_{50} \mathrm{O}$ & 414 & 22.94 & \\
\hline
\end{tabular}

B. courtallica: Barleria courtallica

Table 3: Phytocomponents detected in leaf of $B$. courtallica

\begin{tabular}{|c|c|c|c|c|c|}
\hline RT & Name of the compound & $\begin{array}{l}\text { Molecular } \\
\text { formula }\end{array}$ & $\begin{array}{l}\text { Molecular } \\
\text { weight }\end{array}$ & $\begin{array}{l}\text { Peak area } \\
\%\end{array}$ & Molecular structure \\
\hline 7.87 & $\begin{array}{l}\text { Cyclohexene, 4-ethenyl-4-methyl-3-(1-methylethenyl)- } \\
\text { 1-(1-methylethyl)-, (3R-trans)- }\end{array}$ & $\mathrm{C}_{15} \mathrm{H}_{24}$ & 204 & 0.10 & \\
\hline 8.88 & Caryophyllene & $\mathrm{C}_{15} \mathrm{H}_{24}$ & 204 & 0.07 & \\
\hline
\end{tabular}


Table 3: (Continued...)

\begin{tabular}{ll}
\hline RT & Name of the compound \\
\hline 9.66 & 3-buten-2-one, 4-(2,6,6-trimethyl-1-cyclohexen-1-yl)-
\end{tabular}

10.41

2(3H)-naphthalenone, 4,4a,5,6,7,8-hexahydro-1-methoxy-

13.32 Isoaromadendrene epoxide

14.24 Phytol acetate

17.25 9,12-octadecadienoic acid (Z, Z)-

18.23 9,12-octadecadienoic acid (Z, Z)-, methyl ester

9,12,15-octadecatrienoic acid, 2,3dihydroxypropyl ester, (Z, Z, Z)-
Molecular formula

$\mathrm{C}_{13} \mathrm{H}_{20} \mathrm{O}$

$\mathrm{C}_{11} \mathrm{H}_{16} \mathrm{O}_{2}$

180

0.10

0.07

Peak area $\%$

$\begin{array}{lll}\mathrm{C}_{20} \mathrm{H}_{40} \mathrm{O} & 296 & 34.42 \\ \mathrm{C}_{22} \mathrm{H}_{42} \mathrm{O}_{2} & 338 & 6.53 \\ \mathrm{C}_{18} \mathrm{H}_{32} \mathrm{O}_{2} & 280 & 0.34 \\ \mathrm{C}_{19} \mathrm{H}_{36} \mathrm{O}_{2} & 296 & 0.49\end{array}$

Molecular structure olecular structure<smiles>C/C=C/C(=O)C1=C(C)C=CCC1(C)C</smiles><smiles>COC1=C2CCCCC2CCC1=O</smiles><smiles>C=C(CO)C1CCC2(C)CC(O)C=C(C)C2C1</smiles><smiles>CC1=CCCC(C)C2(CCC(C)(O)C2)C1</smiles><smiles>CC1CCC2CC3C(C4CC5OC5(C)C34)C12</smiles>

3.42

$\mathrm{C}_{20} \mathrm{H}_{40} \mathrm{O} \quad 296$

14.18

$\mathrm{C}_{19} \mathrm{H}_{38} \mathrm{O}_{2} \quad 298$

0.20

$\mathrm{C}_{19} \mathrm{H}_{34} \mathrm{O}_{2} \quad 294$

0.27

$\mathrm{C}_{21} \mathrm{H}_{36} \mathrm{O}_{4} \quad 352$

0.38

$\mathrm{C}_{26} \mathrm{H}_{54}$

366

0.41

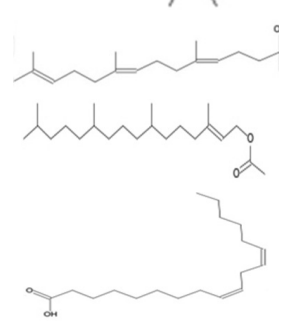

0.54

0.17
$\mathrm{C}_{27} \mathrm{H}_{56} \quad 380$

281

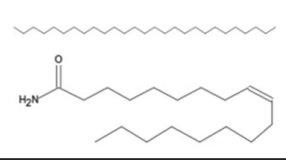


Table 3: (Continued...)

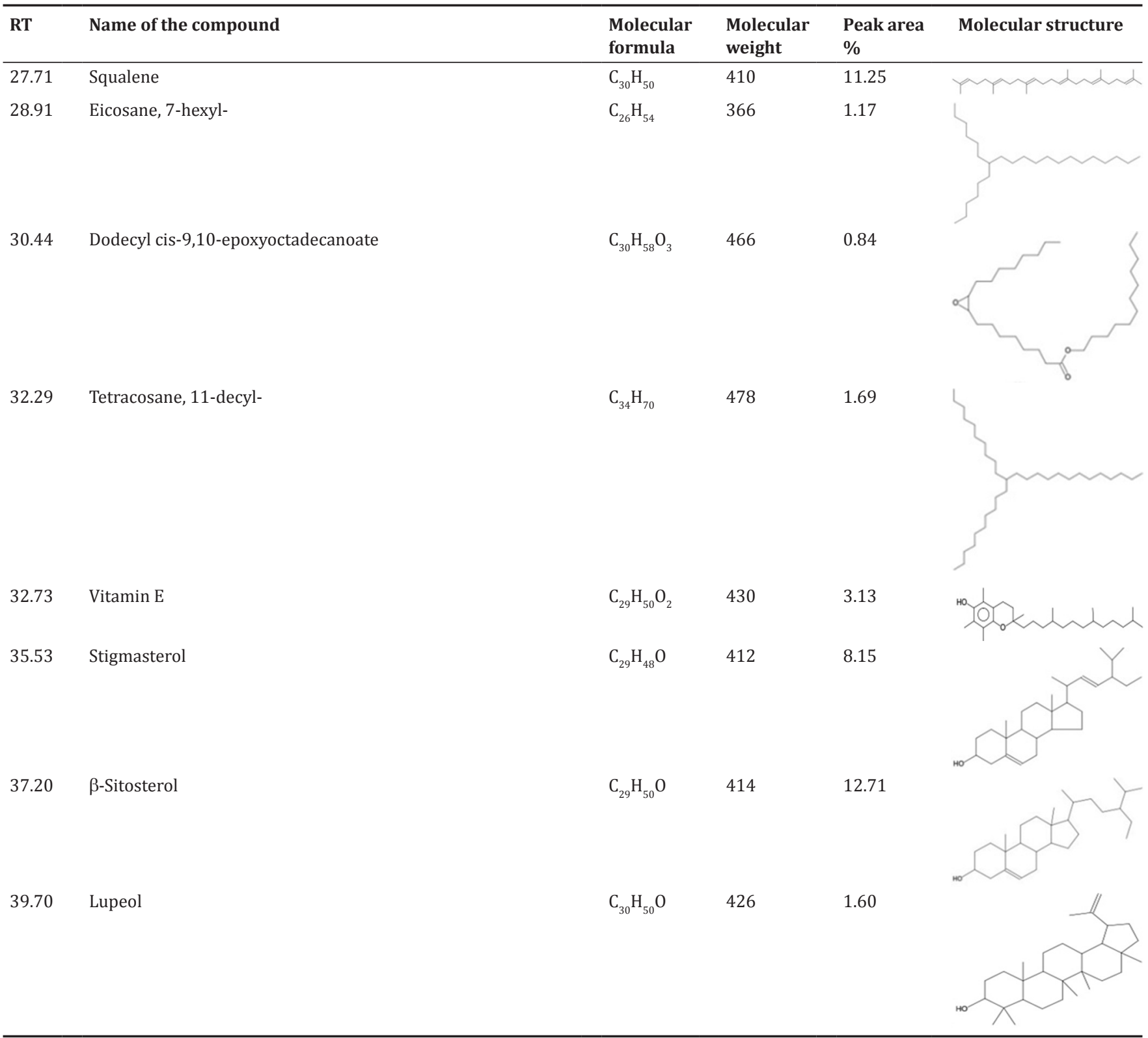

B. courtallica: Barleria courtallica

Table 4: Activity of phytocomponents identified in the ethanol extract of stem, root and leaf of $B$. courtallica

\begin{tabular}{|c|c|c|c|}
\hline Name of the compound & Peak area \% & Compound nature & **Activity \\
\hline p-Cymen-7-ol & 0.12 & Essential oil & $\begin{array}{l}\text { Antimicrobial } \\
\text { Anti-inflammatory } \\
\text { Fragrance }\end{array}$ \\
\hline $\begin{array}{l}\text { 1,4-methanoazulene-9-methanol, } \\
\left.\text { decahydro-4,8,8-trimethyl-, [1S- }\left(1 \alpha, 3 \mathrm{a} \beta, 4 \alpha, 8 \mathrm{a} \beta, 9 \mathrm{R}^{*}\right)\right] \text { - }\end{array}$ & 0.13 & Sesquiterpene alcohol & $\begin{array}{l}\text { Antitumor, Analgesic Antibacterial, } \\
\text { anti-inflammatory sedative, fungicide }\end{array}$ \\
\hline $\begin{array}{l}\text { 4,7,7-trimethylbicyclo[2.2.1]heptan-2-one } \\
\text { 0-allyloxime }\end{array}$ & 0.20 & Nitrogen compound & Antimicrobial \\
\hline Diethyl phthalate & 0.30 & Plasticizer compound & $\begin{array}{l}\text { Antimicrobial } \\
\text { Antifouling }\end{array}$ \\
\hline 3,7,11,15-tetramethyl-2-hexadecen-1-ol & 5.97 & Terpene alcohol & Antimicrobial \\
\hline Phytol, acetate & 1.62 & Diterpene compound & $\begin{array}{l}\text { Antimicrobial } \\
\text { Anti-inflammatory } \\
\text { Anticancer } \\
\text { Diuretic }\end{array}$ \\
\hline Dibutyl phthalate & 5.08 & Plasticizer compound & $\begin{array}{l}\text { Antimicrobial } \\
\text { Anti-inflammatory }\end{array}$ \\
\hline
\end{tabular}


Table 4: (Continued...)

\begin{tabular}{l} 
Name of the compound \\
\hline Phytol \\
9,12-Octadecadienoic acid (Z, Z)-, methyl ester
\end{tabular}

Ethyl Oleate

Curan, 16,17-didehydro-,(20.xi.)-

9-Octadecenamide, (Z)-

Squalene

Vitamin E

Stigmasterol

$\beta$-Sitosterol

Cyclohexene,4-ethenyl-4-methyl-3-(1-methylethenyl)-

1-(1-methylethyl)-, (3R-trans)-

Urs-12-en-28-oic acid, 3-hydroxy-, methyl ester, (3ß)-

Androstane-11,17-dione, 3-[(trimethylsilyl) oxy]-,

17-[0-(phenylmethyl) oxime], $(3 \alpha, 5 \alpha)$ - area $\%$

Compound nature

Linoleic acid ester

Nitrogen compound Amide compound

Triterpene

Vitamin compound

Steroids

Steroids

Sesquiterpene

Ester compound

Steroids
**Activity

Antimicrobial

Anti-inflammatory

Anticancer

Diuretic

Hypocholesterolemic, Nematicide

Antiarthritic

Hepatoprotective

Antiandrogenic, Hypocholesterolemic

5-alpha reductase inhibitor, Antihistaminic Anticoronary

Insectifuge

Antieczemic

Antiacne

Cancer-preventive

Flavor

Hypocholesterolemic

5-Alpha reductase inhibitor

Antiandrogenic

Perfumery

Insectifuge

Anti-inflammatory

Anemiagenic

Dermatitigenic

Choleretic

Antimicrobial

Antimicrobial

Anti-inflammatory

Antibacterial

Antioxidant

Antitumor

Cancer preventive

Immunostimulant

Chemo-preventive

Lipoxygenase-inhibitor

Pesticide

Antitumor antispasmodic Antioxidant

Vasodilator analgesic

Antidiabetic

Hepatoprotective Hypocholesterolemic

Hypoglycemic

Antisterility

Antioxidant

Anti-inflammatory sedative

Antihepatotoxic

Cancer-preventive

Antiviral ovulant

Hypocholesterolemic

Estrogenic artemicide

Antimicrobial

Anti-inflammatory

Anticancer

Antiasthma

Hepatoprotective

Diuretic

Antitumor, analgesic antibacterial,

anti-inflammatory sedative, fungicide

Antimicrobial

Anti-inflammatory

Cytotoxic

Antimicrobial

Anti-inflammatory

Anticancer

Antiasthma

Hepatoprotective

Diuretic 
Table 4: (Continued...)

\begin{tabular}{|c|c|c|c|}
\hline Name of the compound & Peak area \% & Compound nature & **Activity \\
\hline \multirow{6}{*}{$\begin{array}{l}\text { Androstane-11,17-dione, } 3-[(\text { trimethylsilyl)oxy]-, } \\
\text { 17-[0-(phenylmethyl) oxime], }(3 \alpha, 5 \alpha)-\end{array}$} & \multirow[t]{6}{*}{2.89} & \multirow[t]{6}{*}{ Steroids } & Antimicrobial \\
\hline & & & Anti-inflammatory \\
\hline & & & Anticancer \\
\hline & & & Antiasthma \\
\hline & & & Hepatoprotective \\
\hline & & & Diuretic \\
\hline Caryophyllene & 0.07 & Sesquiterpene & $\begin{array}{l}\text { Anti-tumor, analgesic Antibacterial, } \\
\text { anti-inflammatory sedative, fungicide }\end{array}$ \\
\hline \multirow{3}{*}{$\begin{array}{l}\alpha \text {-D-Glucopyranoside, methyl } \\
\text { 2-(acetylamino)-2-deoxy-3-0-cyclic methyl } \\
\text { 7-epi-cis-sesquisabinene hydrate }\end{array}$} & \multirow[t]{2}{*}{0.24} & \multirow[t]{2}{*}{ Amino compound } & Antimicrobial \\
\hline & & & Anti-inflammatory \\
\hline & 0.17 & Sesquiterpene alcohol & $\begin{array}{l}\text { Antitumor, analgesic antibacterial, } \\
\text { anti-inflammatory sedative, fungicide }\end{array}$ \\
\hline Isoaromadendrene epoxide & 0.21 & Sesquiterpene oxide & $\begin{array}{l}\text { Antitumor, analgesic antibacterial, } \\
\text { anti-inflammatory sedative, fungicide }\end{array}$ \\
\hline \multirow[t]{6}{*}{ 9,12-Octadecadienoic acid (Z, Z)- } & \multirow[t]{6}{*}{0.34} & \multirow[t]{6}{*}{ Linoleic acid } & Hypocholesterolemic Nematicide \\
\hline & & & Antiandrogenic Hypocholesterolemic \\
\hline & & & 5-Alpha reductase inhibitor Antihistaminic \\
\hline & & & Anticoronary \\
\hline & & & Insectifuge \\
\hline & & & Antieczemic antiacne \\
\hline \multirow[t]{10}{*}{ 9-Octadecenoic acid (Z)-, methyl ester } & \multirow[t]{10}{*}{0.49} & \multirow[t]{10}{*}{ Oleic acid ester } & Cancer preventive \\
\hline & & & Flavor \\
\hline & & & Hypocholesterolemic \\
\hline & & & 5-Alpha reductase inhibitor \\
\hline & & & Antiandrogenic \\
\hline & & & Perfumery \\
\hline & & & Insectifuge \\
\hline & & & Anti-inflammatory \\
\hline & & & Anemiagenic \\
\hline & & & Dermatitigenic \\
\hline \multirow{7}{*}{$\begin{array}{l}\text { 9,12,15-Octadecatrienoic acid, 2,3-dihydroxypropyl } \\
\text { ester, (Z, Z, Z)- }\end{array}$} & \multirow{7}{*}{0.38} & \multirow{7}{*}{$\begin{array}{l}\text { Linolenic acid ester } \\
\text { compound }\end{array}$} & Antiarthritic \\
\hline & & & Hepatoprotective antiandrogenic \\
\hline & & & Hypocholesterolemic 5-alpha reductase \\
\hline & & & inhibitor Antihistaminic \\
\hline & & & Anticoronary insectifuge \\
\hline & & & Antieczemic \\
\hline & & & Antiacne \\
\hline \multirow{5}{*}{ Lupeol } & \multirow[t]{5}{*}{1.60} & \multirow[t]{5}{*}{ Triterpene } & Antimicrobial \\
\hline & & & Anti-inflammatory \\
\hline & & & Anti-cancer \\
\hline & & & Analgesic \\
\hline & & & Antioxidant \\
\hline
\end{tabular}

B. courtallica: Barleria courtallica, ${ }^{* *}$ activity source: Dr. Duke's phytochemical and ethnobotanical databases

through MS attached with GC. Among the identified phytochemicals, phytol is one among the compounds of the present study. Phytol was observed to have antibacterial activities against Staphylococcus aureus by causing damage in cell membrane; as a result, there is a leakage of potassium ions from bacterial cells [14]. Phytol is a key acyclic diterpene alcohol that is a precursor for vitamin $\mathrm{E}$ and $\mathrm{K}_{1}$. It is used along with simple or corn syrup as a hardener in candies. Phytol is detected in stem, root, and leaf of B. courtallica which was also found to be effective at different stages of arthritis. It was found to possess as well as preventive and therapeutic results against arthritis [15]. Squalene has the property of antioxidant [16]. Recently, squalene possesses chemopreventive activity against colon carcinogenesis [17,18]. Stigmasterol is used as a precursor in the manufacture of semisynthetic progesterone, a valuable human hormone that plays an important physiological role in the regulatory and tissue rebuilding mechanisms related to estrogen effects, as well as acting as an intermediate in the biosynthesis of androgens, estrogens, and corticoids. It is also used as the precursor of vitamin $D_{3}[19,20]$. $\beta$-sitosterol limits the amount of cholesterol absorption in the intestines, therefore decreasing the levels of cholesterol in the body. It is helpful with benign prostatic hyperplasia due to its anti-inflammatory effects and its ability to improve urinary symptoms and flow [21]. Vitamin $\mathrm{E}$ is the main lipid-soluble antioxidant in the body. As antioxidant, vitamin E acts in cell membrane where prevents the propagation of free radical reaction although it has been also shown to have prooxidant activity [22]. Vitamin E supplements may help reduce PMS symptoms, including anxiety, craving, and depression. Vitamin E keeps the skin young by reducing the appearance of fine lines and wrinkles. Vitamin $\mathrm{E}$ is thought to be important chain breaking antioxidant, which plays an important role in various stages of carcinogenesis through its contribution and immunocompetence, membrane and DNA repair, and decreasing oxidative DNA damage [23].

Phthalate, esters of phthalic acid, are mainly applied as plasticizers. Phthalic acid derivatives were suggested to have been used to 
cure chronic cardiovascular and cerebrovascular diseases and had antitumor, anti-inflammatory, and antibacterial functions [24]. Phthalates are reported to have antimicrobial and the pharmacological activities [25]. Lupeol exhibited a broad spectrum of biological activities and can be used as antiprotozoal, anti-inflammatory, antitumor, and chemopreventive agents [26]. The above-said compounds were found in the ethanol extracts of $B$. courtallica which are being used for the pharmacological work.

The presence of various bioactive compounds in the $B$. courtallica justifies the use of whole plant for various ailments. However, isolation of individual phytochemical constituents and subjecting it to the biological activity will definitely give fruitful results. From the results, it could be concluded that $B$. courtallica contains various bioactive compounds. Therefore, it is recommended as a plant of phytopharmaceutical importance.

\section{ACKNOWLEDGMENT}

We would like to thank Dr. S. Kumaravel, Senior Scientist, Department of Food Quality and Testing, Indian Institute of Crop Processing Technology, Thanjavur, for guiding and supporting us throughout.

\section{REFERENCES}

1. Vijayalakshmi R, Ravindran R. Priliminary comparative phytochemical screenning of root extracts of Diospyros ferrea (Wild) Bakh and Aerva lanata (L.) Jass ex. Schultes. Asian J Plant Sci Res 2012;2(5):581-2.

2. Doss A. Preliminary phytochemical screening of some Indian medicinal plants. Anc Sci Life 2009;29:12-6.

3. Pandey P, Mehta R, Upadhyay R. Physico-chemical and preliminary phytochemical screening of Psoralea corylifolia. Arch Appl Sci Res 2013;5(2):261-5

4. Prabhu TP, Panneerselvam P, Atlee WC, Balasubramanium S. GCMS analysis of ethanolic extract of Canthium parviflorum Lamk leaf. J. Appl Pharm Sci 2013;3(2):166-8.

5. Sermakkani M, Thangapandian V. GC-MS analysis of Cassia italica leaf methanol extract. Asian J Pharm Clin Res 2012;5:90-4.

6. Prameela J, Ramakrishnaiah H, Krishna V, Deepalakshmi AP. GC-MS analysis of leaf and root extracts of Didymocarpus tomentosa. Int $\mathrm{J}$ Pharm Pharm Sci 2015;7:423-5.

7. Grover N, Patni V. Phytochemical characterization using various solvent extracts and GC-MS analysis of methanolic extract of Woodfordia fruticose (L.) Kurz. Leaves. Int J Pharm Pharm Sci 2013;4(5):291-5.

8. Saadabi AM, Sehemi AG, AL-Zailaie KA. In vitro antimicrobial activity of some Saudi Arabian plants used in folkoric medicine. Int J Bot 2006;2(2):201-4.
9. Mukherjee PK, Mukherjee D, Maji AK, Rai S, Heinrich M. The sacred lotus (Nelumbo nucifera) - Phytochemical and therapeutic profile. J Pharm Pharmacol 2009;61(4):407-22.

10. Agrawal B, Das S, Pandey A. Boerhavia diffusa Linn.: A review on its phytochemical and pharmacological profile. Asian J Appl Sci 2011;4:663-84

11. Gantait A, Maji A, Barman T, Banerji P, Venkatesh P, Mukherjee PK. Estimation of capsaicin through scanning densitometry and evaluation of different varieties of capsicum in India. Nat Prod Res 2012;26(3):216-22.

12. Singh B, Chandan BK, Prabhakar A, Taneja SC, Singh J, Qazi GN. Chemistry and hepatoprotective activity of an active fraction from Barleria prionitis Linn. in experimental animals. Phytother Res 2005;19(5):391-404.

13. Amoo SO, Finnie JF, Van Staden J. In vitro pharmacological evaluation of three Barleria species. J Ethnopharmacol 2009;121(2):274-7.

14. Inoue Y, Hada T, Shiraishi A, Hirose K, Hamashima H, Kobayashi S. Biphasic effects of geranylgeraniol, teprenone, and phytol on the growth of Staphylococcus aureus. Antimicrob Agents Chemother 2005;49(5):1770-4.

15. Ogunlesi M, Okiei W, Osibole AE. Analysis of the essential oil from the dried leaves of Euphorbia hirta Linn. (Euphorbiaceae), a potential medication for asthma. Afr J Biotechnol 2009;8(24):7042-50.

16. Lalitharani S, Mohan VR, Regini GS, Kalidass C. GC-MS analysis of ethanolic extract of Pothos scandens L. Leaf. J Herb Med Toxicol 2009;3:159-60.

17. Rao CV, Newmark HL, Reddy BS. Chemopreventive effect of squalene on colon cancer. Carcinogenesis 1998;19(2):287-90.

18. Rajeswari G, Muruga M, Mohan VR. GC-MS analysis of bioactive compounds of Hugonia mystax L. Bark (Linaceae). J Pharm Biomed Sci 2013;29(29):818-24

19. Sundararaman P, Djerassi C. A convenient synthesis of progesterone from stigmasterol. J Org Chem 1977;42(22):3633-4.

20. Kametani T, Furuyama H. Synthesis of vitamin D and related compounds. Med Res Rev 1987;7(2):147-71.

21. Rajendrakumar N, Vasantha K, Mohan VR. GC-MS analysis of bioactive components of tubers of Ruellia tuberosa L. (Acanthaceae). Am J Phytomed Clin Ther 2014;2:209-16.

22. Herrera E, Barbas C. Vitamin E: Action, metabolism and perspectives. J Physiol Biochem 2001;57(2):43-56.

23. Machlin LJ, Bendich A. Free radical tissue damage: Protective role of antioxidant nutrients. FASEB J 1987;1(6):441-5.

24. Saeidnia S. Phthalate. In: Wexler P, editor. Encyclopedia of Toxicology. $3^{\text {rd }}$ ed., Vol. 3. London: Elsevier Inc., Academic Press; 2014. p. 928-33.

25. Srinivasan GV, Sharanappa P, Leela NK, Sadashiva GT, Vijayan KK. Chemical composition and antimicrobial activity of the essential oil of Leea indica (Burm. f.). Merr flowers. Nat Prod Rad 2009;8(5):488-93.

26. Gallo MB, Sarachine MJ. Biological activities of lupeol. Int J Biomed Pharm Sci 2009;3:46-66. 